\title{
Immobilisierung, physikalische und elektrochemische Charakterisierung von Gold-Nanopartikeln auf kapazitiven Feldeffekt-Sensoren
}

\author{
Tobias Karschuck $^{1}$, Corinna Kaulen ${ }^{1}$, Arshak Poghossian², Patrick Wagner ${ }^{3}$, Michael J. Schöning ${ }^{1,4}$ \\ 1'Institut für Nano- und Biotechnologien (INB), FH Aachen, Jülich, Deutschland \\ ${ }^{2}$ MicroNanoBio, Düsseldorf, Deutschland \\ ${ }^{3}$ Laboratory for Soft Matter and Biophysics, KU Leuven, Leuven, Belgien \\ ${ }^{4}$ Institut für Biologische Informationsprozesse (IBI-3), Forschungszentrum Jülich GmbH, Jülich, Deutschland \\ Kontakt: karschuck@fh-aachen.de
}

\section{Einleitung}

Gold-Nanopartikel (AuNP) sind durch ihre Biokompatibilität, Stabilität, vielseitige Oberflächenchemie, sowie ihr hohes Verhältnis von Oberfläche zu Volumen für viele biomedizinische Anwendungen attraktiv [1]. Ein aktuelles Beispiel dafür ist ihr Einsatz als optische Marker in Covid-19 AntikörperSchnelltests [2]. Negativ geladene Citrat-stabilisierte AuNP auf der Oberfläche von kapazitiven FeldeffektStrukturen wurden u.a. bereits für die markierungsfreie Detektion von positiv geladenen Makromolekülen, als Nanoträger für Desoxyribonukleinsäure (DNA), die schichtweise Abscheidung von Polymeren und die Immobilisierung von Enzymen verwendet [3].

In dieser Arbeit wird die Immobilisierung von positiv geladenen Aminooctanthiol-stabilisierten GoldNanopartikeln (AuAOT) auf der Oberfläche von kapazitiven Feldeffekt-Sensoren vorgestellt [4]. Die terminale Aminogruppe der eingesetzten Partikel ist sehr gut für die spätere Funktionalisierung mit Biomolekülen geeignet. Die Anbindung der positiv geladenen AuAOTs auf der Sensoroberfläche konnte durch eine signifikante Verschiebung des Sensorsignals nachgewiesen werden. Bei einer höheren Partikeldichte auf der Sensoroberfläche war die gemessene Signaländerung entsprechend ausgeprägter. Zusätzlich konnte die elektrostatische Anbindung des Polyanions Polystyrolsulfonat an der AuAOT-modifizierten Sensoroberfläche gemessen werden.

\section{Methoden und Materialien}

Materialien

Tetrachlorogold(III)-säure-Trihydrat $\left(\mathrm{HAuCl}_{4} \cdot 3 \mathrm{H}_{2} \mathrm{O}\right.$, Thermo Fisher), Trinatriumcitrat-Dihydrat $\left(\mathrm{C}_{6} \mathrm{H}_{5} \mathrm{Na}_{3} \mathrm{O}_{7} \cdot 2 \mathrm{H}_{2} \mathrm{O}\right.$, Merck), Salzsäure $(\mathrm{HCl}, 37 \%$, Merck), Natriumchlorid (NaCl, Merck), Di-Natriumhydrogenphosphat-Dihydrat $\left(\mathrm{Na}_{2} \mathrm{HPO}_{4} \cdot 2 \mathrm{H}_{2} \mathrm{O}\right.$, Merck), Natriumhydroxid (NaOH, Merck), Kaliumchlorid (KCl, Merck), Kaliumdihydrogenphosphat $\left(\mathrm{KH}_{2} \mathrm{PO}_{4}\right.$, Merck), 8-Amino-1-octanthiol- hydrochlorid $\left(\mathrm{C}_{8} \mathrm{H}_{19} \mathrm{NS} \cdot \mathrm{HCl}\right.$, AOT, Merck), Salpetersäure $\left(\mathrm{HNO}_{3}, 68 \%\right.$, Carl Roth), reines Ethanol (99,8\%, Carl Roth) und 4-(2-Hydroxyethyl)-1piperazinethansulfonsäure $\left(\mathrm{C}_{8} \mathrm{H}_{18} \mathrm{~N}_{2} \mathrm{O}_{4} \mathrm{~S}, \mathrm{HEPES}\right.$, Carl Roth) und Fluorwasserstoffsäure (HF, 5\%, AppliChem, Poly(natrium-4-styrolsulfonat) (PSS, Sigma Aldrich) wurden, wie geliefert, verwendet. Alle Glasgeräte wurden vor der Verwendung mit Königswasser gereinigt und mit reichlich Wasser gespült. Ultrareines Wasser (UPW) mit einem spezifischen Widerstand von $14 \mathrm{M} \Omega \cdot \mathrm{cm}$ wurde für alle Experimente verwendet. Siliziumwafer ( $p-S i$, $380 \mu \mathrm{m}$ Dicke, $100 \mathrm{~mm}$ Durchmesser, <100> Orientierung) mit einer $30 \mathrm{~nm}$ dicken Siliziumdioxidschicht $\left(\mathrm{SiO}_{2}\right.$, trockene thermische Oxidation) wurden von Siegert Wafer gekauft.

\section{Synthese der AuNPs}

Citrat-stabilisierte Gold-Nanopartikel (AuCts) mit einem durchschnittlichen Durchmesser von $25 \mathrm{~nm}$ wurden mit der Turkevich-Methode synthetisiert [46]. AuAOTs wurden aus AuCts durch den Ligandenaustausch von Citrat zu Aminooctanthiol hergestellt [4, 7]. Nach mindestens 12 Stunden wurde die AuAOT-Lösung in $1 \mathrm{~mL}$-EppendorfRöhrchen aufgeteilt und durch 15-minütiges Zentrifugieren bei $5000 \mathrm{~g}$ gereinigt, um überschüssiges AOT in der Lösung zu entfernen. Während der Überstand entfernt wurde, blieb das Pellet ungestört und die AuAOTs wurden bei $4{ }^{\circ} \mathrm{C}$ gelagert. Für die Immobilisierungsexperimente wurden die AuAOTPellets mit $250 \mu \mathrm{L}$ eines $20 \mathrm{mM}$ HEPES-Puffers mit $20 \mathrm{mM} \mathrm{NaCl}$ bei pH 3,0 (im Folgenden als Immobilisierungspuffer bezeichnet) redispergiert, um AuAOT-Lösungen mit einer Konzentration von $\mathrm{c}=0,8 \mathrm{nM}$ zu erhalten. Die Partikelkonzentration wurde anhand optischer Extinktionsspektroskopie ermittelt (Jasco-Spektrophotometer V-550, Jasco Labor- und Datentechnik).

Herstellung der kapazitiven Feldeffekt-Strukturen

Die Sensorchips wurden aus handelsüblichen p-SiWafern hergestellt, die mit einer $30 \mathrm{~nm}$ dicken 
thermisch gewachsenen $\mathrm{SiO}_{2}$-Schicht bedeckt waren. Auf der mit Flusssäure geätzten Rückseite des $\mathrm{p}-\mathrm{Si} / \mathrm{SiO}_{2}-$ Wafers wurde durch Elektronenstrahlverdampfung eine Aluminiumschicht (Al, $300 \mathrm{~nm}$ ) abgeschieden. Für eine bessere Haftung zwischen $\mathrm{Al}$ und $\mathrm{p}$-Si wurde der Wafer für $10 \mathrm{~min}$ unter Stickstoffatmosphäre bei $400{ }^{\circ} \mathrm{C}$ getempert. Die $\mathrm{Al} / \mathrm{p}-\mathrm{Si} / \mathrm{SiO}_{2}$-Wafer wurden in $10 \mathrm{~mm} \times 10 \mathrm{~mm}$ große Chips vereinzelt und anschließend in einem Ultraschallbad mit Aceton, Isopropanol, Ethanol und deionisiertem Wasser jeweils für 3 min gereinigt und danach mit $\mathrm{N}_{2}$-Gas bei Raumtemperatur getrocknet.

Die Sensorchips wurden in eine selbstgebaute Messzelle eingebaut, die $1 \mathrm{~mL}$ Elektrolytlösung fasst und eine $0,5 \mathrm{~cm}^{2}$ große Kontaktfläche des Sensorchips mit der Lösung bietet.

Immobilisierung von AuCt- und AuAOTNanopartikeln auf der Sensoroberfläche

Zur Immobilisierung der AuCt-Partikel wurden $40 \mu \mathrm{L}$ AuCt-Lösung auf die $\mathrm{SiO}_{2}$-Oberfläche der gereinigten Sensorchips getropft und bei Raumtemperatur getrocknet. Hinweis: Die AuCt-Nanopartikel wurden nur zur physikalischen Charakterisierung auf den Chipoberflächen immobilisiert.

Die AuAOT-Nanopartikel wurden auf bereits in der Messzelle installierten Sensorchips immobilisiert. Dazu wurden jeweils $40 \mu \mathrm{L}$ der AuAOT-Lösung auf die Sensorchips getropft.

Nach der Immobilisierung von AuCt- oder AuAOTNanopartikeln wurden die Chips mit UPW gespült, in einem sanften $\mathrm{N}_{2}$-Strom getrocknet und mittels Rasterelektronenmikroskopie (REM) untersucht. Jedes Immobilisierungsexperiment wurde mit drei Sensorchips wiederholt.

REM-Bilder der AuNP-modifizierten Sensoroberfläche wurden mit dem JSM-7800F, Jeol, aufgenommen. Der durchschnittliche Partikeldurchmesser wurde an Stellen auf den Chips bestimmt, die eine hohe Anzahl an einzelnen AuNPs aufwiesen. Um die Belegungsdichte der immobilisierten Partikel zu bestimmen wurden 15 Bilder (50.000-fache Vergrößerung) pro modifiziertem Chip aufgenommen. Ein ImageJ-Makro (Version 1.53a, siehe auch [8]) ermöglichte die Analyse der Anzahl und Größe aller Nanopartikel auf jedem REM-Bild.

\section{Elektrochemische Charakterisierung von unmodifizierten und AuAOT-modifizierten Sensorchips}

Abbildung 1 zeigt den schematischen Messaufbau eines AuNP-modifizierten kapazitiven FeldeffektSensors [4]. Für die elektrochemische Charakterisierung wurde der Aluminium-Rückseitenkontakt des Sensorchips und eine in die Elektrolytlösung eingetauchte Referenzelektrode $(\mathrm{Ag} / \mathrm{AgCl}, 3 \mathrm{M} \mathrm{KCl}$ -
Innenelektrolyt; Metrohm) mit einem Impedanzanalysator (Zahner Zennium, Zahner Elektrik) verbunden, um den elektrischen Stromkreis zu schließen.

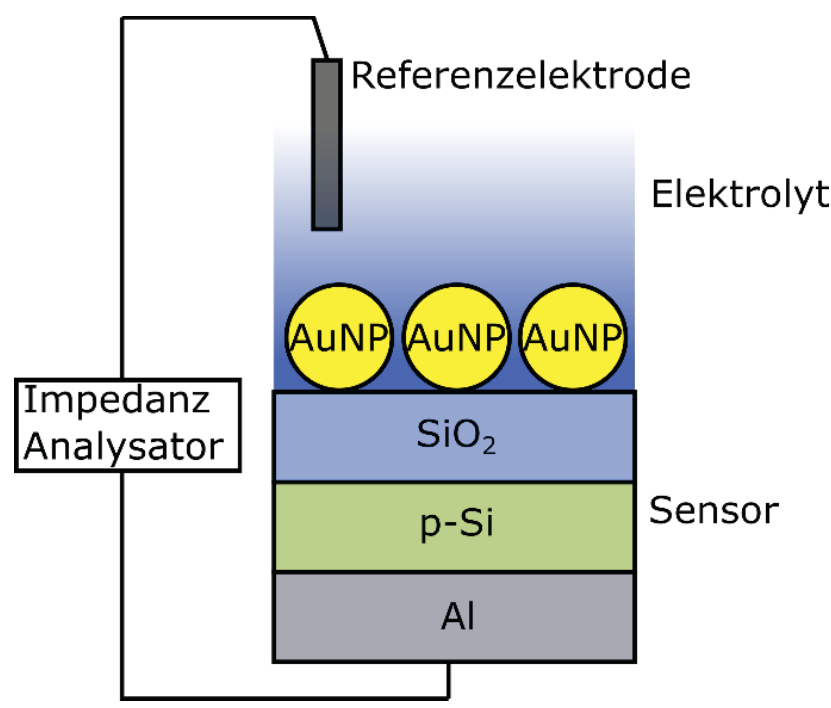

Abb. 1: Schematischer Aufbau eines AuNP-modifizierten kapazitiven Feldeffekt-Sensors. Die Referenzelektrode und der Aluminium-Rückseitenkontakt sind elektrisch mit einem Impedanzanalysator verbunden. (Adaptiert von [4])

Eine ausführliche Erläuterung des Funktionsprinzips des kapazitiven Feldeffekt-Sensors ist an anderer Stelle zu finden [3]. Vereinfacht lässt sich der Sensor als eine Reihenschaltung aus einer konstanten Kapazität (Isolator-SiO $2-S c h i c h t)$ und einer variablen Kapazität der Raumladungszone an der Halbleiter/lsolator-Grenzfläche darstellen. Im Falle einer $\mathrm{Al} / \mathrm{p}-\mathrm{Si} / \mathrm{SiO}_{2}$-Struktur vergrößert sich durch die Immobilisierung der positiv geladenen Nanopartikel die Breite der Raumladungszone, so dass die Gesamtkapazität des Sensors abnimmt. Im ConCap-Modus wird die Kapazität am Arbeitspunkt durch Anlegen einer Regel-Spannung, mit entgegengesetzter Polarität zur Oberflächenpotenzialverschiebung, konstant gehalten. Dies ermöglicht die kontinuierliche Überwachung des Sensorsignals, um Änderungen des Oberflächenpotenzials nachzuweisen. Der Arbeitspunkt wird normalerweise bei etwa $60 \%$ der maximalen Sensorkapazität in der Kapazitäts-Spannungs- (C-V) Kurve ermittelt.

Für die Aufnahme der C-V-Kurve wurde die Kapazität des Sensors in einem Spannungsbereich von $-2 \mathrm{~V}$ bis $2 \mathrm{~V}$ in $100 \mathrm{mV}$-Schritten gemessen. Eine kleine Wechselspannung (Amplitude $20 \mathrm{mV}$, Frequenz $120 \mathrm{~Hz}$ ) wurde für die C-V- und ConCapMessungen überlagert [3]. Alle elektrochemischen Charakterisierungen wurden in einem Faraday'schen Käfig bei Raumtemperatur durchgeführt. 


\section{Ergebnisse}

Die kapazitiven Feldeffekt-Sensoren wurden vor und nach der Immobilisierung der AuAOT-Nanopartikel für 0,5, 1 und 2 Stunden elektrochemisch charakterisiert. Um Störeinflüsse durch Gegenionen zu reduzieren und somit das Sensorsignal zu verstärken, wurden die ConCap-Messungen in einer Pufferlösung mit geringer lonenstärke durchgeführt (0,33 mM PBS (phosphatgepufferte Salzlösung), pH 4, lonenstärke: $5 \mathrm{mM}$ ) [9-12]. Der pH-Wert 4 des Messpuffers wurde gewählt, um sicherzustellen, dass die terminalen Aminogruppen der AuAOTNanopartikel vollständig protoniert sind [7]. Vor der elektrochemischen Charakterisierung wurden die Feldeffekt-Sensoren mindestens 12 Stunden lang in dem Messpuffer konditioniert.

Abbildung 2 zeigt normalisierte ConCap-Messungen von einem unbehandelten Sensor (schwarz), einem AuAOT-modifizierten Sensor (blau) und einem AuAOT-PSS-modifizierten Sensor (rot). In diesem Experiment wurde zunächst die ConCapAntwort des unbehandelten Sensors als Referenzmessung aufgezeichnet. Dann wurden die AuAOTNanopartikel aus einer 0,8 nM AuAOT-Lösung für 0,5 Stunden immobilisiert, gefolgt von einem Spülschritt mit dem Messpuffer, einer Trocknung unter $\mathrm{N}_{2}$-Atmosphäre und einer erneuten elektrochemischen Charakterisierung des AuAOTmodifizierten Sensors. Dieses Vorgehen wurde für weitere $0,5 \mathrm{~h}$ (Gesamtimmobilisierungszeit: $1 \mathrm{~h}$ ) und $1 \mathrm{~h}$ (Gesamtimmobilisierungszeit: $2 \mathrm{~h}$ ) wiederholt, um die Auswirkungen der Immobilisierungszeit auf das Sensorsignal zu untersuchen.

Wie aus Abbildung 2 ersichtlich ist, führt die Immobilisierung von positiv geladenen AuAOTs auf der Sensoroberfläche zu einer Verschiebung des normalisierten ConCap-Signals entlang der Spannungsachse in Richtung negativerer Potenzialwerte. Die Richtung der beobachteten Spannungsverschiebungen entspricht einer stärker positiv geladenen Sensoroberfläche $[3,10,12]$. Die Amplitude der Signalverschiebungen nimmt mit zunehmender AuAOT-Immobilisierungszeit zu und erreicht einen Wert von ca. $-40 \mathrm{mV}$ bei $2 \mathrm{~h}$ Immobilisierungszeit. Diese Signalentwicklung lässt sich durch die Zunahme der Oberflächendichte von AuAOT-Nanopartikeln bei längerer Immobilisierungszeit erklären, welche in Tabelle 1 der mittleren Signaländerung gegenübergestellt wird.

Um die Korrelation zwischen den Sensorsignalen und der AuAOT-Oberflächendichte zu quantifizieren, wurden in separaten Experimenten drei mit AuAOTs bedeckte Sensorchips für die REM-Charakterisierung vorbereitet. Dabei wurden die gleichen Immobilisierungsverfahren wie bei den FeldeffektCharakterisierungen verwendet.

Die Modifizierung von Feldeffekt-Sensoren mit AuNPs stellt eine vielversprechende Plattform für die markierungsfreie Detektion von Biomolekülen dar.

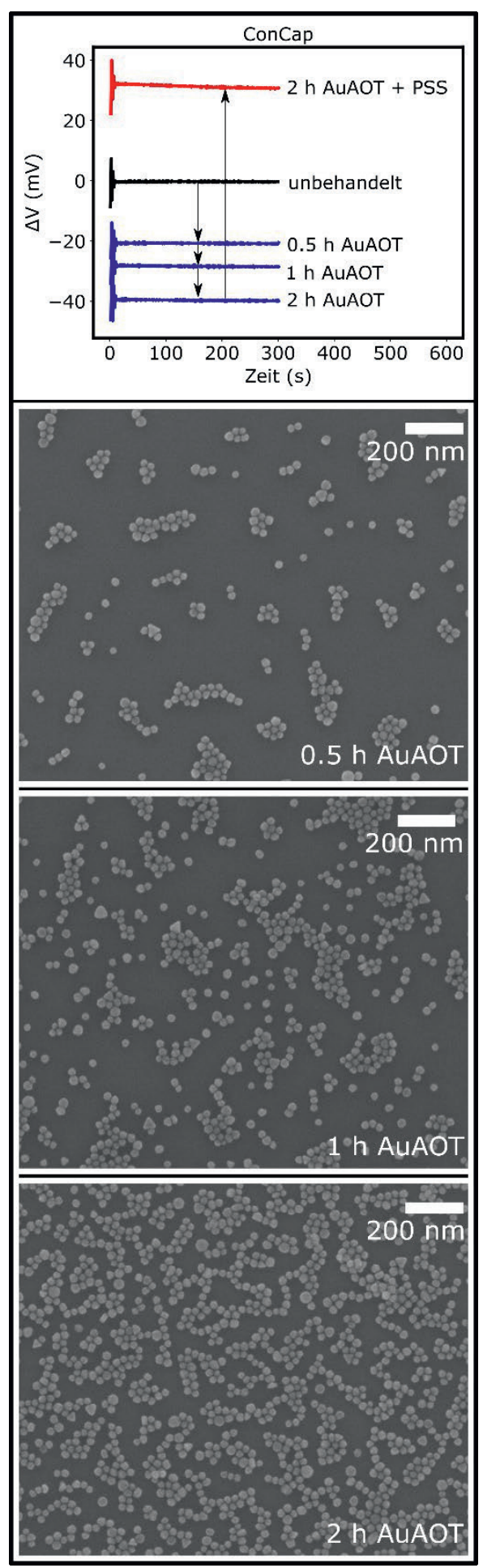

Abb. 2: Die ConCap-Kurve (oben) zeigt die Spannungsänderung aufgrund der Immobilisierung von Aminooctanthiol-stabilisierten (AuAOT) Nanopartikeln auf der Sensoroberfläche nach verschiedenen

Immobilisierungszeiten $(0,5 \mathrm{~h}, 1 \mathrm{~h}, 2 \mathrm{~h})$ in Blau. Die Signaländerung der modifizierten Sensoroberfläche mit Polystyrenesulfonat nach einer zweistündigen AuAOTImmobilisierung ist im Vergleich dazu in Rot dargestellt.

Exemplarische REM-Bilder nach 0,5 h, $1 \mathrm{~h}$ und $2 \mathrm{~h}$

AuAOT-Immobilisierung zeigen die steigende Dichte von AuAOTs mit zunehmender Immobilisierungszeit. 
Tab. 1: Mittlere Signaländerung $(\Delta V)$ mit Standardabweichung $(\mathrm{n}=3)$ der Feldeffekt-Sensoren und Belegungsdichte $(\Gamma)$ nach den unterschiedlichen Immobilisierungszeiten von AuAOTs.

\begin{tabular}{|l|l|l|}
\hline Zeit & $\Delta \mathrm{V}(\mathrm{mV})$ & $\Gamma\left(1 / \mu \mathrm{m}^{2}\right)$ \\
\hline $0,5 \mathrm{~h}$ & $-20,3 \pm 0,6$ & $167 \pm 25$ \\
\hline $1 \mathrm{~h}$ & $-28,7 \pm 1.2$ & $272 \pm 34$ \\
\hline $2 \mathrm{~h}$ & $-39,7 \pm 1.6$ & $540 \pm 86$ \\
\hline
\end{tabular}

In früheren Experimenten wurden beispielsweise mit negativ geladenen AuCts modifizierte FeldeffektSensoren für den markierungsfreien elektrostatischen Nachweis von positiv geladenen Proteinen und Polyelektrolyt-Makromolekülen eingesetzt [13]. Um die Fähigkeit von AuAOTmodifizierten Feldeffekt-Sensoren zur markierungsfreien elektrostatischen Detektion negativ geladener Moleküle zu demonstrieren, haben wir das starke Polyanion Polystyrolsulfonat (PSS) als Modellsystem ausgewählt, da PSS in einem breiten $\mathrm{pH}$ Bereich negativ geladen vorliegt [14].

Abbildung 2 (oben) zeigt auch ein Beispiel für eine ConCap-Messung zum elektrostatischem Nachweis der Kopplung negativ geladener PSS-Moleküle an die Sensoroberfläche, die mit positiv geladenen AuAOT-Nanopartikeln modifiziert wurde. In diesem Experiment wurden die AuAOT-Nanopartikel aus einer 0,8 nM AuAOT-Lösung für $2 \mathrm{~h}$ immobilisiert, gefolgt von einem Spülschritt und der anschließenden Adsorption von PSS-Molekülen. Für die PSSAdsorption wurden $100 \mu \mathrm{L}$ einer $50 \mu \mathrm{M}$ PSS-Lösung (pH 5,4, mit $100 \mathrm{mM} \mathrm{NaCl}$ ) auf die AuAOTmodifizierte Sensoroberfläche getropft und 10 Minuten lang inkubiert. Das ConCap-Signal wurde vor und nach jedem Oberflächen-Modifizierungsschritt im Messpuffer aufgezeichnet.

Die Adsorption negativ geladener PSS-Makromoleküle resultiert in einer Verschiebung des ConCap-Signals um ca. $70 \mathrm{mV}$ in positive Spannungsrichtung, was die erfolgreiche Kopplung der PSS-Makromoleküle an die AuAOT-modifizierte Sensoroberfläche bestätigt.

\section{Diskussion}

Es wurde die schrittweise Immobilisierung von positiv geladenen AuAOT-Nanopartikeln auf kapazitiven Feldeffekt-Sensoren im ConCap-Modus mit variablen Immobilisierungszeiten elektrochemisch untersucht. Zwischen der Oberflächendichte der AuAOTs (bestimmt durch REM) und der gemessenen Potenzialverschiebung der FeldeffektSensoren konnte eine Korrelation ermittelt werden. Je mehr AuAOTs auf der Oberfläche des Sensors immobilisiert wurden, desto deutlicher stieg das Ausgangssignal bei variabler Immobilisierungsdauer an. Die zerstörungsfreie elektrochemische Charakterisierung des AuAOT-Immobilisierungsverfahrens auf Feldeffekt-Sensoren kann als
Prozesskontrolle vor weiteren Oberflächenmodifikationen mit Biomolekülen eingesetzt werden.

Die Fähigkeit der mit AuAOT modifizierten Feldeffekt-Sensoren, die Anbindung negativ geladener Makromoleküle zu erkennen, wurde durch eine zusätzliche Modifikation mit dem Polyanion PSS als Modellsystem für Makromoleküle/Biomoleküle untersucht. Der vorgestellte Ansatz kann für die Funktionalisierung mit Biomolekülen wie z.B. Antikörpern für den Nachweis von Antigenen erweitert werden.

\section{Literatur}

[1] KUMAR, A.; KIM, S.; NAM, J.-M.: Plasmonically engineered nanoprobes for biomedical applications, J. Am. Chem. Soc., 138 (2016), Nr. 44, S. 1450914525

[2] HUANG, C.; WEN, T.; SHI, F.-J.; ZENG, X.-Y.; JIAO, Y.-J.: Rapid detection of IgM antibodies against the SARS-CoV-2 virus via colloidal gold nanoparticlebased lateral-flow assay, ACS Omega, 5 (2020), Nr. 21, S. 12550-12556

[3] POGHOSSIAN, A.; SCHÖNING, M. J.: Capacitive field-effect EIS chemical sensors and biosensors: A status report, Sensors (Basel), 20 (2020), Nr. 19:5639

[4] KARSCHUCK, T.; KAULEN, C.; POGHOSSIAN, A.; WAGNER, P.; SCHÖNING, M. J.: [In Press] Gold nanoparticle-modified capacitive field-effect sensors: Studying the surface density of nanoparticles and coupling of charged polyelectrolyte macromolecules, ELSA

[5] WUITHSCHICK, M.; BIRNBAUM, A.; WITTE, S.; SZTUCKI, M.; VAINIO, U.; PINNA, N.; RADEMANN, K.; EMMERLING, F.; KRAEHNERT, R.; POLTE, J.: Turkevich in new robes: Key questions answered for the most common gold nanoparticle synthesis, ACS Nano, 9 (2015), Nr. 7, S. 7052-7071

[6] POLTE, J.: Fundamental growth principles of colloidal metal nanoparticles - A new perspective, CrystEngComm, 17 (2015), Nr. 36, S. 6809-6830

[7] KAULEN, C.; HOMBERGER, M.; BOURONE, S.; BABAJANI, N.; KARTHÄUSER, S.; BESMEHN, A.; SIMON, U.: Differential adsorption of gold nanoparticles to gold/palladium and platinum surfaces, Langmuir, 30 (2014), Nr. 2, S. 574-583

[8] DOMINEC, F.: Calculating size distribution of powder particles using ImageJ, [Online]. https://www.fzu.cz/ dominecf/index.html, abgerufen am 06.10.2021

[9] LOWE, B. M.; SUN, K.; ZEIMPEKIS, I.; SKYLARIS, C.-K.; GREEN, N. G.: Field-effect sensors - From pH sensing to biosensing: Sensitivity enhancement using streptavidin-biotin as a model system, Analyst, 142 (2017), Nr. 22, S. 4173-4200

[10] BRONDER, T. S.; POGHOSSIAN, A.; JESSING, M. P.; KEUSGEN, M.; SCHÖNING, M. J.: Surface regeneration and reusability of label-free DNA biosensors based on weak polyelectrolyte-modified capacitive field-effect structures, Biosens. Bioelectron., 126 (2019), S. 510-517

[11] HUANG, W.; DIALLO, A. K.; DAILEY, J. L.; BESAR, K.; KATZ, H. E.: Electrochemical processes and mechanistic aspects of field-effect sensors for 
biomolecules, J. Mater. Chem. C, 3 (2015), Nr. 25, S. $6445-6470$

[12] BRONDER, T. S.; POGHOSSIAN, A.; SCHEJA, S.; WU, C.; KEUSGEN, M.; MEWES, D.; SCHÖNING, M. J.: DNA immobilization and hybridization detection by the intrinsic molecular charge using capacitive field-effect sensors modified with a charged weak polyelectrolyte layer, ACS Appl. Mater. Interfaces, 7 (2015), Nr. 36, S. 20068-20075

[13] POGHOSSIAN, A.; BÄCKER, M.; MAYER, D.; SCHÖNING, M. J.: Gating capacitive field-effect sensors by the charge of nanoparticle/molecule hybrids, Nanoscale, 7 (2015), Nr. 3, S. 1023-1031

[14] SMITH, R. N.; MCCORMICK, M.; BARRETT, C. J.; REVEN, L.; SPIESS, H. W.: NMR studies of $\mathrm{PAH} / \mathrm{PSS}$ polyelectrolyte multilayers adsorbed onto silica, Macromolecules, 37 (2004), Nr. 13, S. 48304838

\section{Danksagung}

Teile dieser Arbeit wurden durch die Deutsche Fördergemeinschaft (DFG) - (445454801) gefördert. Die Autoren danken David Rolka und Heiko Iken für die technische Unterstützung. 